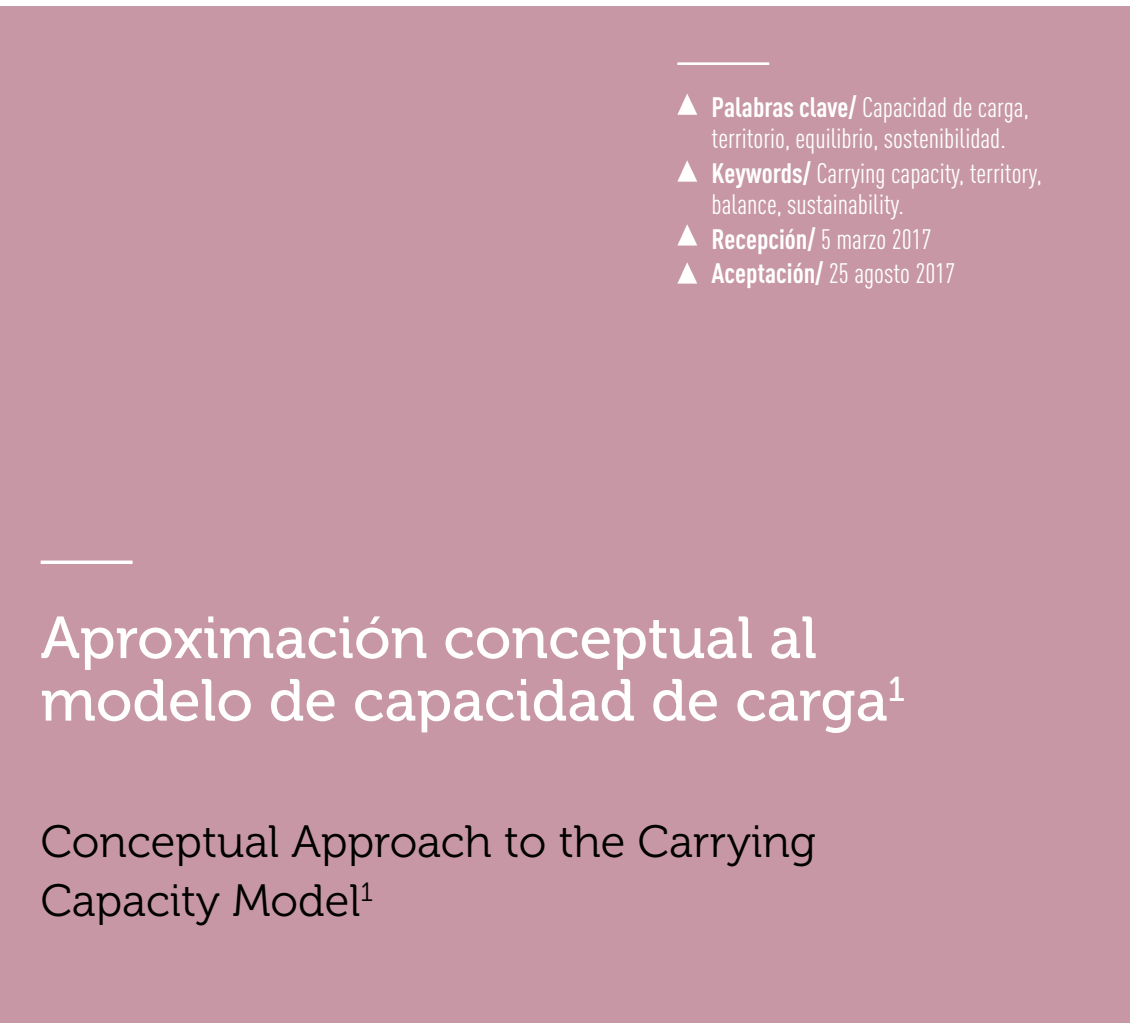

\section{Fabián Sarmiento}

Arquitecto, Universidad Nacional de Colombia Colombia.

Magister en Urbanismo, Universidad Nacional de Colombia, Colombia.

Docente e investigador, Facultad de Arquitectura

Universidad La Gran Colombia, Colombia

Catedrático, Universidad Católica de Colombia y

Pontificia Universidad Javeriana, Colombia.

fabian.sarmiento@ugc.edu.co

\section{Fabián Aguilera}

Arquitecto, Universidad Católica de Colombia, Colombia.

Magíster en Proyectos para el Desarrollo Urbano (IBERO), México.

Docente e investigador, Facultad de Diseño, Universidad Católica de Colombia, Colombia.

faaguilera@ucatolica.edu.co

\section{Juan José Castiblanco}

Arquitecto, Universidad Nacional de Colombia

Colombia.

Magíster en Hábitat, Universidad Nacional de

Colombia, Colombia.

Docente e investigador, Facultad de Diseño,

Universidad Católica de Colombia, Colombia.

juanjosecastiblanco@gmail.com

RESUMEN/ La sostenibilidad de los territorios contemporáneos en Latinoamérica se encuentra en crisis, y el valor de los recursos de nuestra región y su explotación han decantado en efectos negativos para la calidad de vida de nuestro hábitat. Esto ha generado inequidad, pobreza, marginalidad y graves daños a la estructura ambiental. Por estas razones, planteamos la presente investigación desde el paradigma de la sostenibilidad, enfocándonos en la comprensión de las variables que debemos contemplar para cambiar esta lógica auto-destructiva y plantear nuevas estrategias para ordenar sosteniblemente nuestros territorios (Cortés 2010). Este artículo se presenta como una mirada reflexiva, que consolida el concepto de la capacidad de carga como instrumento en la construcción de un modelo incluyente y equitativo para ser aplicado en un futuro en nuestros territorios latinoamericanos. ABSTRACT/ The sustainability of contemporaneous Latin American territories is currently in crisis. The value of resources in our region and their exploitation has resulted in negative impacts for the quality of living of our habitats. This has led to inequality, poverty, marginalization and severe damage to environmental structures. For these reasons, this research is based on the paradigm of sustainability, focusing on the understanding of the variables we need to consider in order to change this selfdestructive rationale and propose new strategies for the sustainable arrangement of our territories (Cortés 2010). This paper is presented as a thoughtful look that consolidates the concept of carrying capacity as a tool to develop an inclusive and equitable approach to be applied in our Latin American territories in the future.

\section{EL TERRITORIO Y LA CAPACIDAD DE CARGA.}

"Se hizo asi evidente que la naturaleza, en un futuro no muy lejano, debe instituir procedimientos de bancarrota contra la civilización industrial..., simplemente como la naturaleza ya ha hecho muchas otras veces a otras especies consumidoras de detritus, con posterioridad a su exuberante expansión y en respuesta a los depósitos de ahorro que sus ecosistemas habian acumulado antes de que tuviesen la oportunidad de comenzar a agotarlos... Habiéndose convertido en una especie súper detritivora, la humanidad quedó destinada no solamente a su sucesión, sino a la quiebra" 2 .

Dentro de los paradigmas contemporáneos, la sostenibilidad es esencial para la cualificación de la calidad de vida. Sin embargo, en el contexto latinoamericano, solo hace poco tiempo comenzamos a reflexionar sobre elementos técnicos que permitan aplicarlo en la gestión de nuestro territorio. Universidad Católica de Colombia (Bogotá Colombia).

76 'This paper was prepared under the cross-institutional research titled "Sustainable Re-densification Strategy in Urban Borders", conducted by Universidad La Gran Colombia and Universidad Católica de Colombia (Bogota, Colombia). 
Medina y Bermúdez plantean la problemática en América Latina, indicando que "[e]/ crecimiento del espacio urbano, sobre todo en los países en vías de desarrollo, ha sido desordenado e incontrolable, y su resultado es el predominio de ciudades fragmentadas, caóticas, dispersas, congestionadas y ambientalmente insostenibles, que favorecen la segregación, el anonimato y la individualidad" (Medina y Bermúdez 2010: 73). Dicha premisa se enfoca ambiental, social y económicamente en los bordes interurbanos y periurbanos que han generado un modelo inequitativo en Latinoamérica (figura 1). La migración del campo, la concentración de la riqueza, el deterioro de recursos básicos y la afectación de la productividad, evidencian la crisis del modelo de ocupación del territorio a lo largo de los siglos XX y XXI.

En este escenario, cabe preguntarse cuáles son los retos que deben asumirse para hacer sustentable el crecimiento latinoamericano. Asimismo, muchos aspectos merecen ser considerados, en cuanto afectan la supervivencia de nuestra biósfera y biodiversidad. Por esta razón, el presente texto se enfoca en desarrollar el concepto de capacidad de carga, entendiéndolo como la relación entre el hábitat humano y el modelo económico que direcciona la construcción del territorio a partir de los recursos naturales de los que el ser humano dispone para su desarrollo.

Capacidad de carga es un concepto proveniente de las ciencias naturales y determina atributos o cualidades de un ecosistema que le permiten no colapsar mientras la población que lo habita presenta un crecimiento (Morales 2011) (figura 2). Platón, refiriéndose a la capacidad de soportar las necesidades de la población a escala local y global, afirmaba: "el territorio que era anteriormente suficiente para alimentar a la gente no será ya suficiente, sino pequeño" (1988: 128). En la actualidad, elementos como los tratados comerciales, cambio climático, concentración y aumento de población, deterioro de recursos -por mencionar algunos- generan pobreza, inequidad, discriminación y aumento de conflictos por recursos.

Así, la sostenibilidad se plantea como una alternativa que, ligada a la capacidad de carga, posibilita el análisis y proyección de los recursos para soportar las actividades de la población, garantizando capitales ambientales, sociales y económicos en un modelo equilibrio. Por lo anterior, plantearemos la capacidad de carga como

\section{APROXIMACIÓN METODOLÓGICA.}

En este trabajo se plantean dos estadios metodológicos. El primero abarca la comprensión de los elementos que definen nuestro concepto de capacidad de carga y sus implicaciones. El segundo, incluye el desarrollo de una matriz de variables que posibilite la discusión de indicadores temáticos en las tres dimensiones de la sostenibilidad -lo social, lo económico y lo ambiental- aportando una base de desarrollo para su posterior aplicación en

espacios académicos y de gestión urban DESDE LA CAPACIDAD DE CARGA.

El territorio se define, idealmente. por dinámicas de equidad, eficiencia, estabilidad, sostenibilidad y calidad de vida. En el contexto de esta investigación, se consideran dos elementos cuyo estudio en profundidad es esencial para plantear este modelo: la población y los recursos (figura 3).

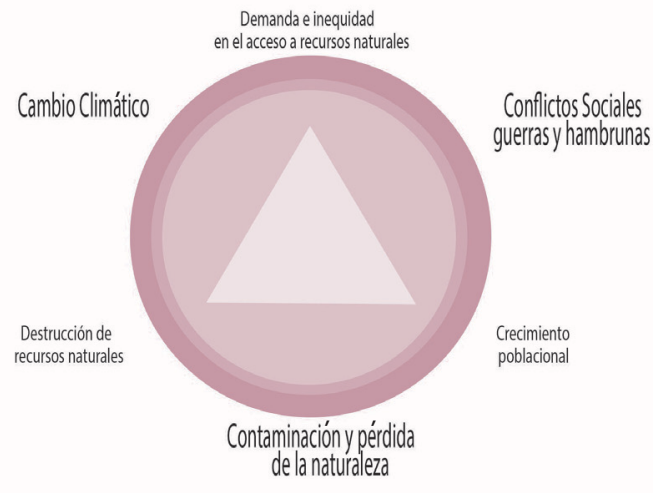

Figura 1. Génesis de la Ecocrisis (fuente: El autor).

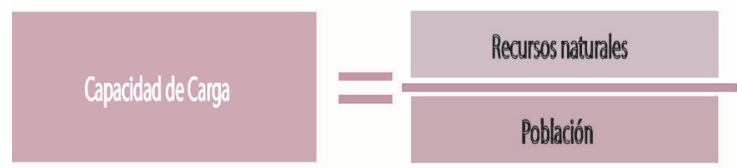

Figura 2. Definición de la ecuación de la capacidad de carga, según Jean Pierre Morales Aymerich (fuente: El autor).

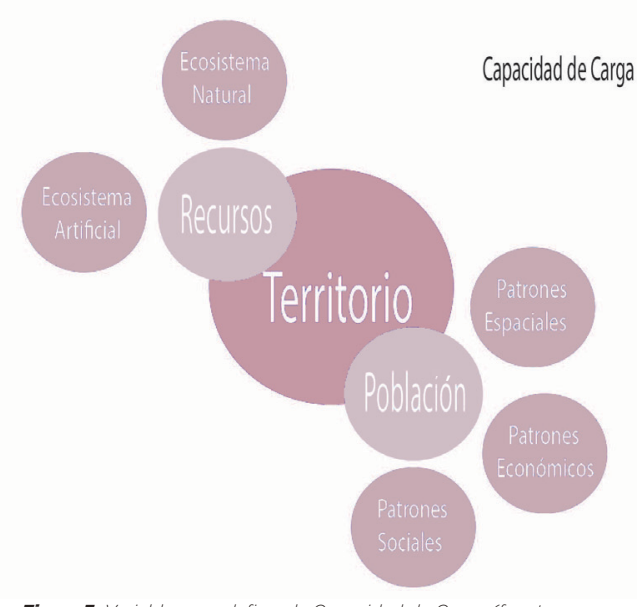
Figura 3. Variables que definen la Capacidad de Carga (fuente:
El autor). 


\section{LA POBLACIÓN Y SU EFECTO EN EL TERRITORIO.}

Desde la teoría de la ecología y el medio ambiente, la población es un conjunto de seres vivos que moran en el territorio y se apropian de él. Existen dos variables relacionadas a la población: la primera, es el número de individuos que la conforman y la segunda, el impacto que ésta genera en su hábitat, esto visto desde la teoría de la dependencia de recursos de Pfeffer y Salancik (1978). En el caso del ser humano, se trata de una especia única, ya que su número no se reduce por falta de recursos sino que construye redes globales para consumir recursos de otros territorios. Desde la economía, es posible afirmar que la población es el motor del desarrollo. A mayor población, mayor capacidad de crecimiento, lo que genera consecuencias diferenciales. Por un lado, impulsa la plusvalía y, por otro, el efecto del avance de la economía desde la reserva adicional de mano de obra, reduce costos y aumenta las ganancias para pocos, tal como lo evidencia Marx (2009).

Finalmente, desde lo social, la población es un sistema de patrones que se encuentra vinculado a los elementos del territorio y la cultura, de manera que la población transforma su entorno y sus relaciones con la estructura natural y el espacio (Alexander 1977)

Así, planteamos la hipótesis de que la población es responsable del consumo y transformación de recursos que permiten su existencia, y, por lo tanto, es responsable de gestionar y sostener el territorio (Lewis 1957), rompiendo con la teoría del crecimiento de Hoselitz (1961), que nos ha llevado a la crisis consumista actual. Desde la variable de la población, encontramos dos dinámicas. La primera es el crecimiento como estrategia de conquista, consolidando el fenómeno urbano como proceso de control, sin reparar en consecuencias como la baja calidad del hábitat y el crecimiento desmesurado de la población mundial y latinoamericana (tabla 1)

\begin{tabular}{|c|c|}
\hline AÑO & POBLACIÓN MUNDIAL \\
\hline 1960 & 3,034 \\
\hline 1965 & 3,325 \\
\hline 1970 & 3,685 \\
\hline 1975 & 4,066 \\
\hline 1980 & 4,438 \\
\hline 1985 & 4,843 \\
\hline 1990 & 5,285 \\
\hline 1995 & 5,71 \\
\hline 2000 & 6,118 \\
\hline 2005 & 6,517 \\
\hline 2010 & 6,931 \\
\hline 2015 & 7,355 \\
\hline
\end{tabular}

POBLACIÓN LATINOAMERICA

Nota: Las cantidades se expresan en miles de millones

Tabla 1. Crecimiento poblacional mundial y latinoamericano (fuente: El autor en base a datos del Banco Mundial).

El segundo fenómeno es la aglomeración. Según Ascher (1995), las aglomeraciones son territorios ligados a dinámicas poblacionales con relación a las actividades del territorio. Esta mirada se fortalece en Gravagnuolo (1998) y la historia de la ciudad desde la ocupación del suelo, categorizando las aglomeraciones según las escalas de impacto sobre el suelo: pueblo, ciudad metrópoli, megalópoli, región y metapoli. Cada definición está ligada a las cantidades de población, procesos de ocupación del suelo y redes socio culturales, caracterizando las condiciones de relación locales y globales, y la relación entre recursos y población, que potencia espacios críticos como los bordes en los territorios. La aglomeración es consecuencia del crecimiento producido por fenómenos como la migración, la búsqueda de oportunidades y la calidad de vida que anhelan los habitantes de los territorios de borde, los cuales plantean problemáticas de informalidad, marginalidad y exclusión social en la aglomeración urbana.

Los efectos críticos en la aglomeración urbana contemporánea han sido generados por el crecimiento poblacional y la demanda sin control de recursos para su sustento. Esta situación ha decantado en impactos negativos sobre los territorios, afectando los espacios geográficos locales y globales que potencian modelos de ocupación extensivos. Éstos, a su vez, deterioran de manera directa los recursos naturales que posibilitan nuestro sustento, tales como e

\section{LOS RECURSOS Y EL LÍMITE DE NUESTROS TERRITORIOS.}

Hemos definido, desde los conceptos planteado previamente, el valor de la población como variable en los modelos de sostenibilidad. Ahora nos concentraremos en el territorio como objeto impactado y su relación con los recursos. Al revisar los modelos propuestos por el urbanismo en la historia latinoamericana, se evidencia su relación con la localización, dominio, gestión 


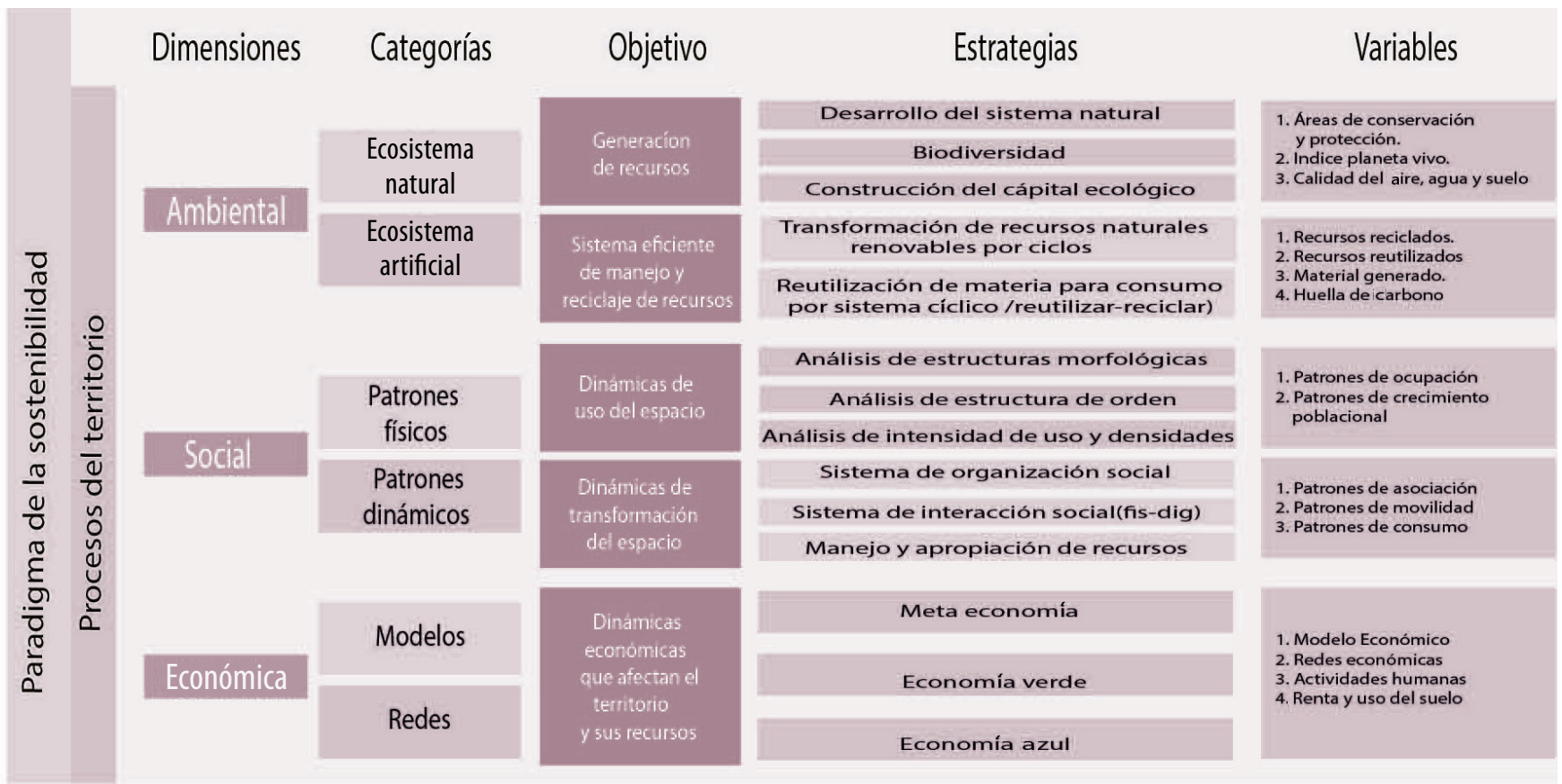

Figura 4. Definición conceptual de la variable de recursos para un modelo sostenible (fuente: El autor).

y administración de los recursos.

La relación con los recursos naturales es tan importante que para la fundación de ciudades era esencial la presencia de agua potable, recursos alimentarios y material para la construcción de las edificaciones. Esto es tangible en el modelo de leyes de indias aplicado a ciudades como Bogotá,

Quito o Lima, por mencionar algunos

territorios latinoamericanos.

Pero, ¿qué son los recursos? El derecho ambiental los define como

"[a]quellos componentes de la naturaleza susceptibles de aprovechamiento para la satisfacción de las necesidades humanas y que presentan un valor -actual o potencial-, donde se destacan cuatro características básicas. Primero, es un componente natural que cumple condiciones para ser reconocido. Segundo, es aprovechable porque conocemos su potencial. Tercero, su aprovechamiento está orientado a satisfacer necesidades. Cuarto, presenta valor actual o potencial según la necesidad a la que se destine" (Andaluz 2006: 41-42).
Sin embargo, esta definición es insatisfactoria en el contexto del paradigma de la sostenibilidad, ya que responde al insostenible modelo consumista. Así, replanteamos la definición desde la metaeconomía, la economía verde y la economía azul. Para comenzar, son componentes de la naturaleza que el hombre debe comprender en todas sus dimensiones para hacer uso adecuado de ellos y garantizar la sostenibilidad de los ciclos de la naturaleza. Además, su aprovechamiento está vinculado al conocimiento de los ciclos para favorecerlos dentro de la naturaleza. Asimismo, su aprovechamiento está orientado a la satisfacción de las necesidades de los ecosistemas y territorios para garantizar la calidad de vida de los ecosistemas de los que el hombre hace parte. Finalmente, el valor de cualquier recurso está ligado al beneficio de la misma naturaleza de la cual hace parte el hombre, vinculando los capitales ambientales, económicos y sociales en su correcta gestión y administración.
La ecocrisis nos plantea la relación nociva del hombre con la naturaleza y muestra la situación de conflicto que vive el mundo, la cual favorece intereses económicos sobre recursos básicos para nuestra sobrevivencia, tal como lo destaca el "Informe de planeta vivo de 2016", donde se evidencian los daños que hemos generado a la biodiversidad debido al interés económico de explotación de monocultivos y la monocría de animales

\section{(WWF International 2016).}

\section{EN BUSCA DE UN NUEVO MODELO PARA ENTENDER LA CAPACIDAD DE CARGA.}

Para plantear un modelo conceptual que responda a la capacidad de carga, hemos construido una matriz que articula definiciones teóricas desde el paradigma de la sostenibilidad. Esta estructura se ordena por dimensiones, categorías, objetivos, sistema de estrategias y variables, instrumentalizando el modelo o estrategia de ordenamiento del territorio (figura 4). 


\section{LA DIMENSIÓN AMBIENTAL.}

Esta dimensión se enfoca en la producción, gestión y administración de recursos a partir de los ecosistemas que lo conforman, definiendo dos categorías. La primera es el ecosistema natural, que es la estructura del territorio natural, y el manejo autónomo de sus ciclos de renovación y transformación de materia, garantizando biodiversidad y capital ambiental (Banos-González, Martínez-Fernández y Esteve-Selma 2015). Para la comprensión integral de esta dimensión, se han definido tres conceptos: el espacio geográfico, que corresponde al recurso de suelo, se caracteriza por finito y lo llamamos territorio; el índice de planeta vivo, que define los valores de la diversidad biológica de un espacio geográfico (Cortés 2009) y que construye equilibrios entre las especies para su sostenibilidad; y, por último, los recursos naturales de soporte. que son aquellos que posibilitan la existencia y el funcionamiento de los ecosistemas $y$, por ende, de las actividades humanas. La segunda categoría es el ecosistema artificial, que se compone de espacios geográficos domesticados por la acción humana para su beneficio. Éstos se evalúan desde los ciclos ecológicos de reutilización y reciclaje con acciones para transformar y vincular ciclos cerrados que reduzcan el impacto en ecosistemas naturales. Se valoran a través de cuatro variables: la primera es el proceso de reciclado; la segunda, los recursos reutilizados; la tercera, es la materia que se transforma para el ciclo económico y como estrategias de manejo hasta entrar de nuevo en un nuevo ciclo (reutilización de materia orgánica, de agua, uso de $\mathrm{CO}_{2}$ como materia, entre otros); finalmente, se plantea la evaluación de la huella de carbono aplicada a procesos, reutilización y reciclaje.

\section{DIMENSIÓN SOCIAL.}

Esta dimensión se enfoca en las dinámicas propias de la sociedad, ligadas a procesos sostenibles, planteándose dos categorías. La primera es la construcción de patrones para comprender efectos en el territorio, considerando la relación de los patrones de ocupación (modelos compactos o dispersos), y los patrones de crecimiento poblacional (que dan razón de las fuerzas que tensionan los recursos y el espacio). La segunda es la construcción de los patrones dinámicos, cuyo propósito es entender la complejidad de la sociedad (Banos-González et al. 2015) y fenómenos intangibles, como la organización social, los patrones de movilidad de personas o bienes y la relación de la población y los recursos. Este patrón está compuesto de tres niveles: el patrón de asociación (construcción de dinámicas de integración social, como grupos de arte, comunidades por origen, etc.); el segundo es el patrón de movilidad, que cuestiona la interacción de bienes y personas desde la compacidad urbana; y el último es la comprensión de los patrones de consumo, que vinculan las redes sociales y culturales propias del lugar.

\section{DIMENSIÓN ECONÓMICA.}

Esta dimensión está ligada a los modos de transformación de los recursos al servicio de las actividades humanas desde dos categorías: una es el modelo económico que potencia o precisa las reglas de relación entre población y recursos; la segunda es la construcción de redes que posibilitan el funcionamiento del modelo económico y que son tangibles en el territorio (infraestructura, tejidos urbanos y rurales, edificabilidad y densidad, entre otros)

Para evaluar esta dimensión, se plantean criterios desde la meta-economía, la economía verde y la economía azul. Éstas son cuatro variables: la primera es la evaluación del modelo económico desde la relación población y recursos; la segunda es la revisión de redes para entender procesos de jerarquización, impacto y transformación del territorio; la tercera es la evaluación de actividades humanas por efecto de la tecnología y el consumo en el modo de vida; la cuarta es la renta y uso del suelo en relación a los valores del capital económico, ambiental y social, a través de la apropiación y la manera de proteger los recursos y compromisos de la sociedad con el cambio de paradigma.
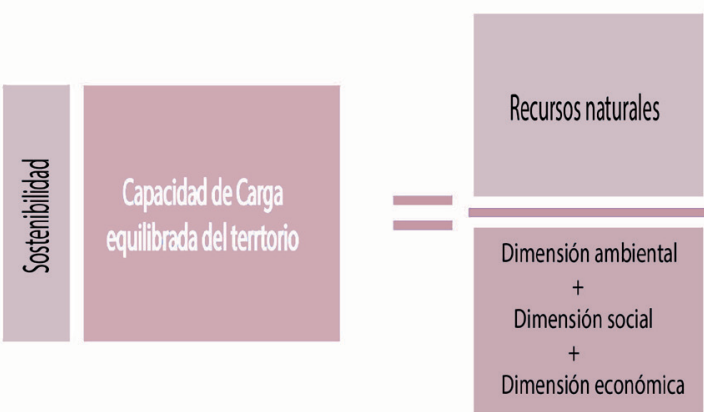

Figura 5. Síntesis de la ecuación de evaluación para un modelo sostenible (fuente: El autor). 
Este planteamiento nos permite concluir que es necesaria la modificación de la ecuación original que define la capacidad de carga, integrando las dimensiones ambiental, social y económica como parte del impacto sobre los recursos y los territorios, planteando un esquema multidimensional y multidisciplinar para afrontar los retos de la ecocrisis (figura 5). Es necesario, de igual manera, presentar retos desde la complejidad, para vincular las dinámicas de procesos enfocados en el cambio de los modelos de relación con nuestro entorno natural y así proyectar una capacidad de carga en equilibrio.

Para garantizar un futuro sostenible de nuestras sociedades y nuestros procesos, será necesario comprender y proyectar variables de forma estructural en el consumo de los recursos y la gestión de la materia, facilitando ciclos que enlacen la reutilización y reciclaje dentro de un sistema basado en el modelo de desarrollo y consolidación de dinámicas de equidad, integración y calidad de vida para los ecosistemas que requiere nuestra sociedad latinoamericana. $\mathbf{\Delta}$

\section{REFERENCIAS}

Alexander, C., 1977. A pattern language: towns, buildings, construction. Berkeley: Oxford University Press. Andaluz, C., 2006. Manual de derecho ambiental. Primera edición. Lima: Edición Proterra. Ascher, F., 1995. Métapolis: ou l'avenir dês villes. París: Editorial Odile Jacob.

Banos-González, I., Martinez-Fernández, J. y Esteve-Selma, M., 2015. "Dynamic integra-tion of sustainability indicators in insular socio-ecological systems." Ecological Modelling, 306, (2015): 130-144. Cortés, S., 2009. "La capacidad de carga como herramienta para la ordenación sostenible del territorio." Boletín CF+S, 42-43, s/p. Disponible en: http://habitat.aq.upm.es/boletin/n42/aa-scor.html Gravagnuolo, B., 1998. Historia del urbanismo en Europa 1750-1960 (Vol. 14). Madrid: Ediciones Akal. Hoselitz, B. (Ed.), 1961. Theories of economic growth. California: Free Press.

Lewis, W., 1957. "Teoría del desarrollo económico." El Trimestre Económico, 96 (4): 454-467.
Marx, K., 2009. El Capital. Critica de la economía politica, el proceso de producción del capital. Vigésimocuarta reimpresion. Ciudad de Mexico: Siglo XXI Editores.

Medina. P. y Bermúdez, D., 2010. "La sostenibilidad urbana en Colombia." Revista Bitácora, 17, 73-93. Morales, J., 2011. "La capacidad de carga: conceptos y usos." Recursos Naturales y Ambiente, 63, 47-53. Pfeffer, J. y Salancik, G., 1978. The Externa/ Control of Organizations. Stanford: Stanford University Press. Platón, 1988. Diálogos VI: República. Madrid: Editorial Gredos S.A.

The International Society for Ecological Economics e Island Press, 1994. " Invirtiendo en capital natural: una aproximación a la sostenibilidad ecológica. " Disponible en: https://www.crisisenergetica.org/staticpages/ capacidad_carga.htm

WWF International, 2016. Planeta vivo, Informe 2016. Disponible en: http://wwf.panda.org/es/noticias_y_ publicaciones/publicaciones/informe_planeta_vivo_2016/ 